\title{
Proactive or Reactive Routing: A Unified Analytical Framework in MANETs
}

\author{
Xianren Wu, Hui Xu, Hamid R. Sadjadpour and J.J. Garcia-Luna-Aceves
}

\begin{abstract}
We present a mathematical framework for the performance evaluation of proactive and reactive routing protocols operating in mobile ad hoc networks (MANETs). The model captures the functionality of the routing protocols together with the characterization of the performance of the medium access control protocol (MAC). It reveals the interplay between the protocol functionality and network parameters, and provides new insight on the relative benefits of proactive and on-demand routing in MANETS. The analytical results are corroborated with results obtained using discrete-event simulations.
\end{abstract}

\section{INTRODUCTION}

The two main classes of routing protocols for mobile ad hoc networks (MANETs) are proactive and reactive (or ondemand). Proactive routing protocols provide fast responses to topology changes by maintaining routing information for all network destinations and react to changes in the network. However, the price to pay is the signaling overhead incurred in maintaining routing information for those destinations in which large numbers of nodes have no interest. On the other hand, reactive routing protocols provide routing information on a need-to-have basis and, at least in theory, can reduce the signaling overhead incurred in maintaining routing tables compared to proactive approaches. However, on-demand routing may incur long setup times in discovering the routes to new destinations.

Given that proactive and reactive routing in MANETs have relative advantages and disadvantages, comparing the two is important. Significant work (e.g., [1], [2], [3], [4], [5], [6], [7]) has been conducted to evaluate and compare these protocols under network profiles of various mobility and traffic configurations. Such performance comparisons have been mostly conducted via discrete-event simulations. Simulationbased studies of routing schemes is indeed a powerful tool to gain insight on their performance for specific choices of network parameters. However, it is difficult to draw conclusions involving multidimensional parameter spaces, because running several simulation experiments for many combinations of network parameters is impractical.

Few if any analytical studies have been pursued on this topic, and has been mostly restricted to the analysis and comparison of routing control overhead [8], [9]. Zhou et al. [8] present an analytical view of routing overhead of reactive protocols, assuming a static Manhattan grid network and study the scalability of reactive protocols. Viennot et al. [9] proposed parametric models for proactive and reactive protocols to evaluate their individual routing control overheads. None of these works evaluates the effects of signaling overhead on unicast capacity at nodes, and neither of them reveals the underlying connection between protocol performance and network parameters.

Given that previous work does not establish an analytical connection between protocol performance (e.g. packet delivery ratio and delay) and network parameters (e.g. node density, mobility and traffic density), analytical models are needed to characterize and compare the performance of routing protocols as a function of the characteristics of the physical layer, the operation of the underlying MAC protocol, and the mobility of nodes. This paper proposes a general, parameterized framework for analyzing protocol performance in mobile ad-hoc networks. In our framework, the adverse effects of signaling overhead on data packets are captured and analyzed through a two-customer queuing model of the operation of nodes. The framework is a combinatorial model that parameterizes and evaluates the performance of routing protocols using a joint characterization of the routing and channel access functionalities in terms of packet delivery ratio and delay. This model focuses on the essential behavior of on-demand and proactive routing protocols, rather than on specific routing protocols. However, when tailored to specific protocol, the proposed model gives good approximations to simulated protocol performance with the IEEE 802.11 MAC using the Distributed Coordination Function (DCF), further corroborating its effectiveness and correctness in dealing with protocol performance in more realistic scenarios.

Section II presents the mobility model, traffic model and simplified models of routing algorithms used in Section III to model the performance of proactive and on-demand routing in MANETs. Section IV characterizes the performance of MAC protocols based on scheduling (TDMA) and contention (802.11 DCF). Section V compares our analytical results against extensive Qualnet simulations based on scenarios using various traffic loads, mobility and node density configurations. The results indicate that our analytical framework provides a good first-order approximation of the performance of MANET routing protocols, and that it can predict the impact of various network parameters analytically, which can then be followed by a simulation-based study focusing on concrete parameter values. Section VI concludes this paper.

\section{NETWORK MODEL}

For convenience, we first present a brief summary of parameters used throughout the paper, as well as their short descriptions in Table I. In the network, nodes are assumed to be mobile and to be uniformly distributed over the network initially. The movement of each node is independent and 
TABLE I

SUMMARY OF PARAMETERS

\begin{tabular}{|l|l|}
\hline$N$ & Number of nodes \\
$\delta$ & Node density \\
$V$ & Average nodes' speed \\
$R$ & Radius of transmission circle \\
$F$ & Number of parallel traffic flows in the network \\
$K$ & Average hop-count per source-destination pair \\
$\lambda_{B}$ & Mean broadcast flooding rate \\
$T_{L}$ & Average link lifetime \\
$C$ & Effective unicast capacity \\
$\rho$ & Signaling efficiency \\
$C_{u}$ & Unicast capacity per node \\
\hline
\end{tabular}

unrestricted, i.e, the trajectories of nodes can lead to anywhere in the network. For node $i \in\{1,2, \ldots, N\}$, let $\left\{T_{i}(t), t \geq 0\right\}$ be the random process representing its trajectory and take values in $D$, where $D$ denotes the domain across which the given node moves. To simplify our model, we make the following assumption on the trajectory processes.

Assumption 1: [Stationarity] Each of the trajectory processes $\left(T_{i}(t)\right)$ is stationary and the $N$ trajectory processes are jointly stationary.

The above assumption implies that the spatial node distribution reaches its steady-state distribution irrespective of the initial location, and that the entire network eventually reaches the same steady state from any initial node placements, within which the statistical spatial nodes' distribution of the network remains the same over time. This lays the foundation for the modeling of node movement. Most existing models, (e.g., random direction mobility models [10], random waypoint mobility models [11], [12] and random trip mobility model [13]) clearly satisfy our assumption. In other words, our assumption ensures that, in the long run, the network converges to its steady state and the stationary spatial nodes' distribution can be used in the performance analysis of the network.

We consider a new traffic flow, which we also call a new session, as one that is associated by the arrival of a new application-level session request at a node $i$ with some destination $j, j \neq i$ in the network. Traffic flows are randomly generated with uniformly distributed sources and destinations. In this work, we assume long-lived traffic flows in order to investigate protocol performance under steady state of node mobility and traffic distributions. Short-lived traffic flows, reflecting transient behaviors, are beyond the scope of the paper.

We assume that the network topology is well connected. More precisely, if an existing path for any traffic session is broken, then with high probability there is an alternative path available to support the continuing operation of the traffic flow. The alternative path is not necessarily disjoint with the former broken path.

We assume the following generic behavior of proactive and reactive routing protocols, which we believe capture the essential behavior of many designs and implementations of routing protocols. However, this analysis, and hence the generic protocols below, does not consider many protocolspecific techniques aimed at improving the efficiency with which protocols operate, such as multi-point relays, local repairs, and route caching mechanisms.

Proactive Routing Protocol: Every node maintains a list of destinations and their routes by processing periodic topology broadcasts originated by each node in the network. When a packet arrives, the node checks its routing table and forwards the packet accordingly. Each node monitors its neighboring links and every change in connectivity with any neighbor results in a topology broadcast packet that is flooded over the entire network. In a well-connected network, the same topology broadcast packet could reach nodes multiple times and therefore enjoy a good packet reception probability. In this paper, we assume that every node receives topology flooding packets reliably from other nodes.

Reactive Routing Protocol: Nodes maintain their routing tables on a need-to-use basis. This implies that, when a new traffic session arrives, nodes have to set up the path between the source and destination before data packets can be forwarded. The path-setup process is called route discovery. Node $i$ initiates this process upon the arrival of a "new traffic session" in order to discover a new path to a node $j$. To accomplish this, node $i$ floods the whole network with route request (RREQ) packets searching for a route to destination $j$. Upon receiving the RREQ packet, node $j$ sends out a route reply (RREP) packet along the reverse path to $i$. A route maintenance process is necessary to find alternative paths if existing paths are broken. A node $i$ is informed that a link along an active path has broken, such that it can no longer reach the destination node $j$ through that route. Upon reception of a notification of a route failure, node $i$ can initiate a route discovery again to find a new route for the remaining packets destined to $j$.

\section{UNIFIED FRAMEWORK FOR QUANTIFYING PROTOCOL PERFORMANCE}

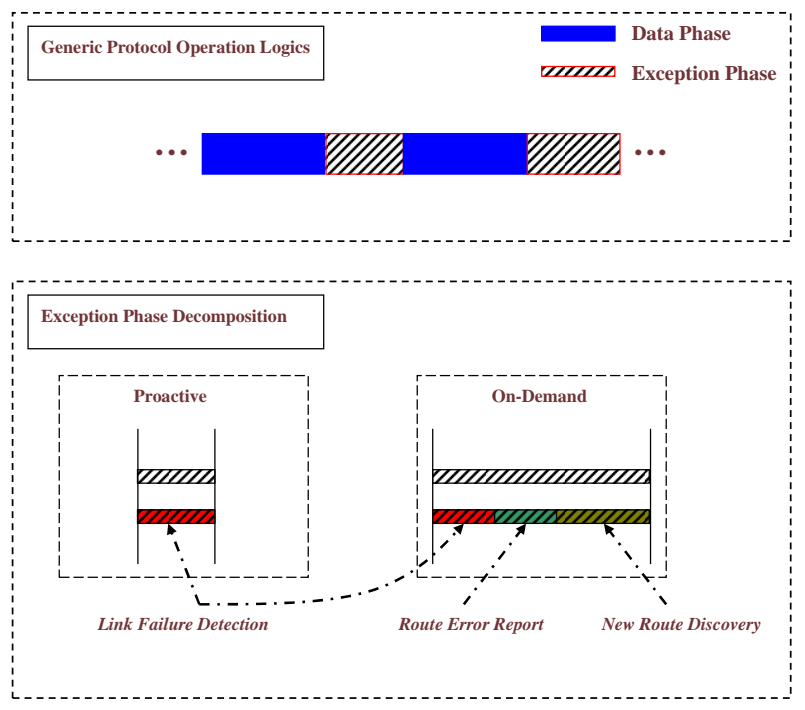

Fig. 1. Generic Protocol Operation

In general, protocol performance should be the convolving result from protocol design philosophy and MAC performance at nodes. Bearing distinctive design philosophies, proactive 
and reactive protocols exhibit dramatic performance difference. Furthermore, signaling overhead changes significantly with different designs and in turn result in significant MAC performance variations. To evaluate the performance of a protocol, we start with an analytical characterization of signaling overhead in terms of mean broadcast flooding rate $\lambda_{B}$. We then bring out a combinatorial model with two parameters: signaling efficiency $\rho$, capturing the generic effect from design philosophy; and unicast capacity $C_{u}$, measuring the MAC performance in handling unicast packets as well as reflecting the adverse effects from signaling overheads. These two parameters are then synthesized to produce the overall performance measure of protocol performance - effective unicast capacity $C$. Mathematically, the model can be written as,

$$
C=\rho \times C_{u}
$$

Nevertheless, Eq.(1) is a rather simple model for characterizing protocol performance, leaving out many nuances in protocol behaviors. However, this simple model captures essential aspects of routing protocols, accounting for the complex interplay from protocol designs and MAC. Network parameters, such as node density, traffic, and mobility, are embedded in the model and their contributions will be analytically exploited, as we move on evaluating the model.

\section{A. Mean Broadcast Flooding Rate $\lambda_{B}$ : Characterization of Signaling Overhead}

Clearly, a mean broadcast flooding rate $\lambda_{B}$ that reflects routing overhead plays an essential role in determining protocol performance. Generating such flooding packets is directly connected with stability of topology. Knowledge of stability of topology can be applied to compute the mean broadcast flooding rate [14]. In our generic protocols, we assume that every topology change, mostly from nodes' mobility, triggers a broadcast flood event.

We know that a topology is comprised of the set of all active links participating in the protocol operation and it usually involves with significant number of active links. Let the set of all active links be denoted by $A_{s}(t)$ and $N_{s}(t)=\left|A_{s}(t)\right|$ be the number of links in the active set, where $|\cdot|$ is the cardinality operator and $t$ is the time index. Note that the topology changes with time $t$ and due to the ergodicity in the joint trajectory processes, its stationary distribution can be derived from the stationary spatial nodes' distribution with respect to the underlying mobility models[14].

When a network is running in steady-state and the process of topology change is ergodic, it will experience all possible topologies with an associated probability vector derived from the steady-state nodes' distribution. By averaging all possible topologies, we can compute complementary cumulative distribution function (CCDF) $F(t)$ characterizing the stability of topology [14] as

$$
F(t) \approx \exp \left(-E\left(N_{s}(t)\right) * t / T_{L}\right) .
$$

It should be pointed out that only the breakage process of existing links are counted in the above analysis, while formation process of new links is not included. However, in proactive protocols such as the optimized link state routing (OLSR) protocol [15], both the formation and breakage process should be taken into account, because both of them could trigger protocol events. Luckily, in the long run, for a network with finite number of nodes, the formation and breakage process should be balanced off each other. Then the overall CCDF distribution accounting for both the formation and breakage process will be

$$
F(t) \approx \exp \left(2 \times-E\left(N_{s}(t)\right) * t / T_{L}\right)
$$

It is also worthy to note that, for reactive protocols such as ad hoc on-demand distance vector (AODV) routing [16], only the breakage process will trigger the protocol event and the stability of topology should be evaluated by Eq.(2).

Summarizing the analysis, we can approximate the mean broadcast rate as

$$
\lambda_{B}= \begin{cases}E\left(N_{s}(t)\right) / T_{L}, & \text { reactive } \\ 2 E\left(N_{s}(t)\right) / T_{L} . & \text { proactive }\end{cases}
$$

For reactive protocols, $E\left(N_{s}(t)\right)$ can be approximated as,

$$
E\left(N_{s}(t)\right) \approx K * F .
$$

And for proactive protocols, $E\left(N_{s}(t)\right)$ can be approximated as [14],

$$
E\left(N_{s}(t)\right) \approx C_{N}^{2} *\left(\pi R^{2} * \delta\right)
$$

\section{B. Signaling Efficiency $\rho$ : Reflections on Protocol Design Philosophy}

We first parameterize the operation of a routing protocol focusing on a given traffic flow, say from node $i$ to node $j$. Because we are interested in long-term behavior with steady traffic, the initial traffic and network setup cost are usually negligible.

As illustrated in Fig. 1, the operation of the traffic flow can be generally classified into two alternating phases: a data phase and an exception phase. During a data phase, an active path to a destination has been established and data packets are forwarded from node $i$ to $j$ along the active route. An exception phase is triggered when a link failure is detected in an active path and an alternative path needs to be discovered. Let $T_{a}$ and $T_{e}$ be the mean duration of a data phase and exception phase, respectively. And let signaling efficiency $\rho$ be the ratio between the data phase and the overall time.

$$
\rho=T_{a} /\left(T_{a}+T_{e}\right)
$$

Both proactive and reactive protocols share similar data phases, because they are determined by the underlying joint trajectory processes for nodes. Therefore, one parameter $T_{a}$ is used for both protocols. However, the time for exception phase is quite different. As depicted in Fig. 1, further decomposition of an exception phase reveals that proactive and reactive protocols bear different behaviors. The exception phase $T_{e}^{p}$ in proactive protocols involves only the time window $W_{l}$ which is a protocol parameter for link failure detection, i.e,

$$
T_{e}^{p}=W_{l}
$$


For reactive protocols, the exception phase $T_{e}^{o}$ involves four steps:

- Link failure detection, denoted by $W_{l}$.

- Link failure unicasted back to source, denoted by $T_{l f}$.

- RREQ broadcast flooding, denoted by $T_{r r e q}$.

- RREP unicasted back to source, denoted by $T_{r r e p}$.

From this decomposition, we have

$$
T_{e}^{o}=W_{l}+T_{l f}+T_{r r e q}+T_{r r e p}
$$

The signaling efficiency $\rho^{p}$ (or $\rho^{r}$ ) of a generic proactive protocol (or reactive protocol) can then be evaluated as,

$$
\begin{aligned}
\rho^{p} & =T_{a} /\left(T_{a}+W_{l}\right) \\
\rho^{r} & =T_{a} /\left(T_{a}+W_{l}+T_{l f}+T_{\text {rreq }}+T_{\text {rrep }}\right)
\end{aligned}
$$

For now, the routing signaling can be represented by a tuple of parameters called signaling parameter tuple (SPT) $\vec{\theta}_{s}=$ $\left\{T_{a}, W_{l}, T_{l f}, T_{\text {rreq }}, T_{\text {rrep }}\right\}$.

\section{Unicast Capacity $C_{u}$ : Reflection on MAC performance}

During a data phase, data packets are unicasted along the active path from a source to the destination. From a queuing perspective, nodes along the active path form a tandem network of queues. Given that every node takes two types of traffic (broadcast packets and unicast packets), each node can be modeled as a two-customer queue. To simplify the analysis, we make the following assumptions for the queuing model:

- The nominal packet length is $L$ for both broadcast and unicast packets, while the model can be extended to incorporate various packet length distributions.

- The arrival process of broadcast (or unicast) traffic is Poisson with parameter $\lambda_{B}$ (or $\lambda_{U}$ ). Such a Markovianinput assumption can be justified theoretically as the sum of a large number of independent random traffic flows from the neighboring nodes. Each node is now modeled as a M/G/1 FCFS queue.

- Every queue operates independently of any other. This is a strong hypothesis in our analysis, because the traffic among nodes may be heavily correlated, especially when data traffic between nodes originates from one same source rather than multiple independent streams. However, in practice, the model still gives a very satisfactory approximation, as observed from simulation results reported in [17].

Each node can now be represented by a tuple of parameters called MAC parameter tuple (MPT) $\vec{\theta}_{m}=\left\{\lambda_{B}, \lambda_{U}, \bar{S}_{B}, \bar{S}_{U}, \mathcal{V}_{B}, \mathcal{V}_{U}, P_{e}\right\}$, where $\left\{\bar{S}_{B}, \mathcal{V}_{B}\right\}$ (or $\left\{\bar{S}_{U}, \mathcal{V}_{U}\right\}$ ) stand for the mean and variance of service time of broadcast packets (or unicast packets) respectively and $P_{e}$ denotes the packet loss probability.

Knowing MPT, we can evaluate the unicast capacity $C_{u}$ as,

$$
C_{u}=E\left(\left(1-\lambda_{B} \bar{S}_{B}\right) \frac{1}{\bar{S}_{U}}\right)
$$

Clearly, proactive (or reactive) protocols enjoy their individual unicast capacity $C_{u}^{p}$ (or $C_{u}^{r}$ ), because they exhibit different
MAC performance, mostly induced from different signaling overhead $\lambda_{B}$.

Eq. (12) implies a significant constraint on network scalability. Specifically, to ensure protocols operating at correct logics, nodes performing the task of delivering packets should be functional. Since nodes are modeled as M/G/1 queues, for queues to be stable and functional, we can infer the scalability constraint [18] as,

$$
E\left(\lambda_{B} \bar{S}_{B}+\lambda_{U} \bar{S}_{U}\right)<1
$$

The left side of the equation is a function of network size $N$.

\section{Delay Aspect \& Packet Delivery Ratio}

From the two-customer M/G/1 model, we can compute the one-hop delay of broadcast packets $D_{B}$ or unicast packets $D_{U}$ as [18]

$$
\begin{aligned}
D_{B} & =\bar{S}_{B}+\frac{\lambda_{B}\left(\bar{S}^{2}{ }_{B}+\mathcal{V}_{B}\right)+\lambda_{U}\left(\bar{S}^{2}{ }_{U}+\mathcal{V}_{U}\right)}{2\left(1-\lambda_{B} \bar{S}_{B}-\lambda_{U} \bar{S}_{U}\right)} \\
D_{U} & =\bar{S}_{U}+\frac{\lambda_{B}\left(\bar{S}^{2}{ }_{B}+\mathcal{V}_{B}\right)+\lambda_{U}\left(\bar{S}^{2}{ }_{U}+\mathcal{V}_{U}\right)}{2\left(1-\lambda_{B} \bar{S}_{B}-\lambda_{U} \bar{S}_{U}\right)}
\end{aligned}
$$

Since nodes are randomly moving under an ergodic process, the active path could experience all possible source-destination distributions and on the long run, the mean end-to-end delay $D_{p}$ can be computed as

$$
\begin{aligned}
D_{p} & \approx K \times D_{U}+D_{b u f} \\
D_{b u f} & \approx(1-\rho) * T_{e} / 2
\end{aligned}
$$

where $D_{b u f}$ specifies the average delay for packets stored in buffer during the exception phase.

The end-to-end packet delivery ratio (PDR) $P_{d}$ can be approximated as

$$
P_{d} \approx\left(1-P_{e}\right)^{K}
$$

\section{E. Evaluation of Signaling Parameter Tuple}

In SPT $\vec{\theta}_{s}, T_{a}$ measures the average path lifetime and can be approximated as $T_{a} \approx T_{L} / K . T_{L}$ usually takes the form as $T_{L}=\Theta(R / V)$ [19] and can be written as,

$$
T_{L}=c 1 * R / V
$$

where $c 1$ is a constant determined from the underlying mobility model. $T_{l f}$ is the average time of RRER packets traveling back to the source. Since the path can break at any point in the middle and if assumed uniform distribution of such breakages, it is computed as

$$
T_{l f}=K / 2 * D_{U}
$$

$T_{\text {rreq }}$ denotes the average time of broadcast packets from source to reach destinations and can be written as

$$
T_{\text {rreq }}=K * D_{B}
$$

$T_{\text {rrep }}$ denotes the average time of RREP packets delivered back to sources and is derived as

$$
T_{\text {rrep }}=K * D_{U}
$$




\section{MAC PARAmeter Tuple: Characterizing MAC PERFORMANCE}

The only remaining question consists of characterizing the performance of the MAC protocol, reflected in MAC parameter tuple $\vec{\theta}_{m}=\left\{\bar{S}_{B}, \bar{S}_{U}, \mathcal{V}_{B}, \mathcal{V}_{U}, p_{e}\right\}$, which we do next. Particularly, we consider three representative MAC schemes. One is the global time division multiple access (GTDMA [20]), which serves as a lower achievable bound. The second one is also a TDMA scheme, but the scheduler is locally optimal (LTDMA [21]). In practice, no such schedulers are used, because instant global topology information is required and the design of any such scheduler is known to be an NP-hard problem. However, such a scheme serves the purpose of an upper performance bound for scheduled MAC protocols. Finally, we consider the widely deployed contention-based MAC scheme, 802.11 DCF MAC, which we aim at characterizing more practical protocol analysis.

\section{A. Global Time Division Multiple Access}

In the GTDMA scheme, the channel access of nodes is organized as frames in time and each frame is further organized into $N$ slots. In every frame, every node in the network is assigned a slot for transmission and the duration of slot should allow nodes to transmit the maximum transmission unit (MTU).

Let's $\Delta_{g}$ be the duration of a slot and the duration of a framework will be $\Delta_{f}=N \Delta_{g}$. In such fashion, every node will get one slot to sent out one packet (either broadcast packet or unicast packet) for every $\Delta_{f}$ time. During the scheduled access, there will be no collision in packet transmission and thus it is safe to assume that the packet loss probability will be zero, i.e, ${ }^{1}$

$$
P_{e}=0
$$

It is also clear that every node enjoys a deterministic service time of $\Delta_{f}$. For such special case, M/G/1 model is thus reduced to a two-customer M/D/1 model. Correspondingly, one have

$$
\begin{aligned}
& \mathcal{V}_{B}=\mathcal{V}_{U}=0 \\
& \bar{S}_{B}=\bar{S}_{U}=\Delta_{f}
\end{aligned}
$$

\section{B. Local Genie-TDMA}

In contrast to GTDMA, LTDMA is a localized TDMA scheme where the transmission of nodes are scheduled locally. For node $i$, if it has $N_{r}-1$ neighbors, the channel access is still grouped as frames but each frame has only $N_{r}$ slots for all $N_{r}$ nodes, who are within coverage of nodes $i$. However, the design of such a scheduling scheme for all nodes without collisions is sometimes impossible or an NP problem. We assume that there is always one such Genie-scheduler and the results obtained serve as an upper bound on performance.

For such a scheme, the packet loss probability is also zero

$$
P_{e}=0
$$

${ }^{1}$ Note that we don't consider wireless environmental effects, e.g. fading, conforming to the well-known protocol model [22].

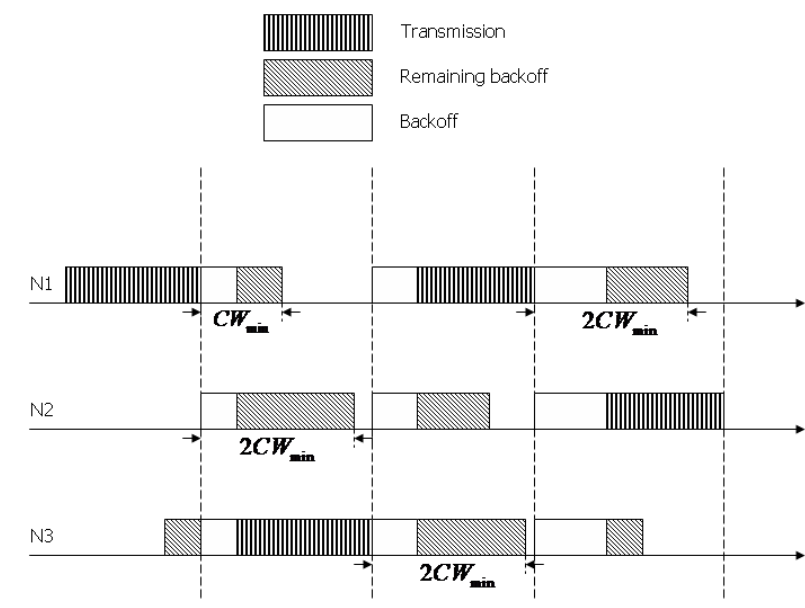

Fig. 2. CSMA/CA sketch.

However, the service time now becomes,

$$
\begin{aligned}
& \mathcal{V}_{B}=\mathcal{V}_{U}=\Delta_{g}^{2} \operatorname{Var}\left(N_{r}\right) \\
& \bar{S}_{B}=\bar{S}_{U}=\Delta_{g} E\left(N_{r}\right)
\end{aligned}
$$

where $\Delta_{g}$ denotes the time duration of a slot and $\operatorname{Var}(\cdot)$ is the variance operator of a random variable. Clearly, $N_{r}$ is a random variable characterizing the statistical distributions of the number of nodes in a communication circle. If distributions of nodes are uniform, $N_{r}$ will be binomial distributed as

$$
\begin{aligned}
P\left(N_{r}=K\right) & =C_{N}^{K} p^{K}(1-p)^{N-K} \\
p & =\pi R^{2} \times \delta / N
\end{aligned}
$$

where $p$ is the probability of two nodes being within communication range of each other. Then, we have

$$
\begin{aligned}
E\left(N_{r}\right) & =N p \\
\operatorname{Var}\left(N_{r}\right) & =N p(1-p)
\end{aligned}
$$

\section{Contention-based MAC}

We consider the well-known 802.11 DCF MAC, employing carrier sense multiple access with collision avoidance (CSMA/CA) technique. In such a scheme, broadcast packets and unicast packets are processed differently and will therefore have different service time.

For unicast packets, a rotating back-off mechanism is adopted to resolve contention. The whole procedure is illustrated in Fig. 2. For the first transmission of a packet, if the channel is sensed to be idle for an interval greater than Distributed Inter-Frame Space (DIFS), the node initializes a backoff timer. And the value of the backoff timer is uniformly selected within the initial contention window (CW) $C W_{\min }$. The timer decrements when the channel is sensed to be idle, freezes when the channel becomes busy and restart when the channel becomes idle for a DIFS again. When the timer counts down to zero, packet is transmitted immediately and waits for an acknowledgment (ACK) confirmation. In case that an ACK is not received and the last transmission is declared a failure, the value of $\mathrm{CW}$ is doubled for retransmission, until it reaches the upper limit of $C W_{\max }$ specified by the protocol. 


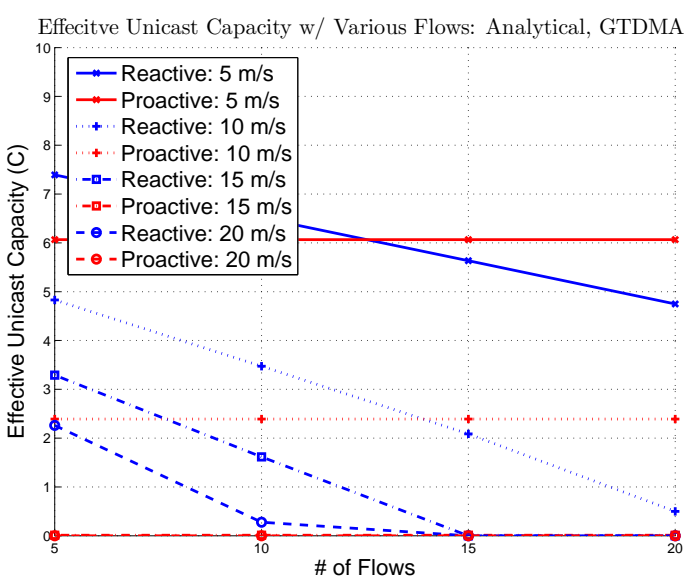

Fig. 3. Effective Unicast Capacity, Various Flows: Analytical, GTDMA.

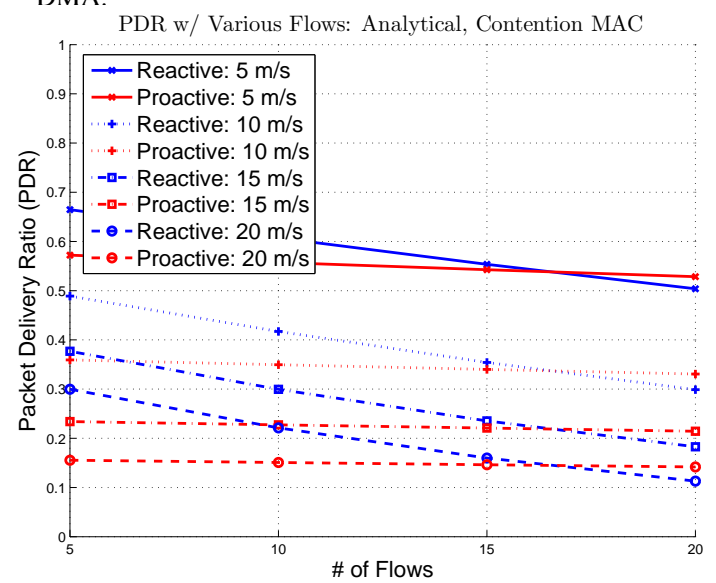

Fig. 5. Packet Delivery Ratio (PDR), Various Flows: Analytical, Contention-based MAC

For broadcast packets, no retransmission are attempted and no ACK is needed. Each broadcast packet is transmitted only once. Therefore, broadcast packets only need to go through the first trial phase of unicast packet transmission, i.e., the phase with the initial contention window of $C W_{\min }$.

To analyze the MAC performance of a node $i$, we first look at its probability generating function $C_{i}(z)$ of channel occupancy, as observed from node $i$. Channel occupancy of node $i$ is used to characterize the distribution of channel utilizations from its neighboring nodes. $C_{i}(z)$ employs a generic representation form as $C_{i}(z)=\sum_{n} P\left(C_{i}=n\right) z^{n+1}$, where $C_{i}$ is expressed in discretized slot duration, $P\left(C_{i}=n\right)$ denotes the probability of channel being sensed as busy for a continuous period of $n$ slots and $z$ is a dummy variable. Such discretized slot representation may introduce some small deviations. However, because the slot duration $\eta$ is usually a very small value, such discretization effect could be neglected.

Clearly, the identity channel generating function $C_{i}(z)=$ $p\left(C_{i}=0\right) z=z$ would mean that $n=0$ always, i.e., the channel is permanently sensed idle by node $i$. We assume that all packets sent to the channel are of the same length $L$. Therefore, there are only two kinds of channel status: idle because of no packet arrival and busy because of some arrival with packet length $L$. In this case, we can simplify the

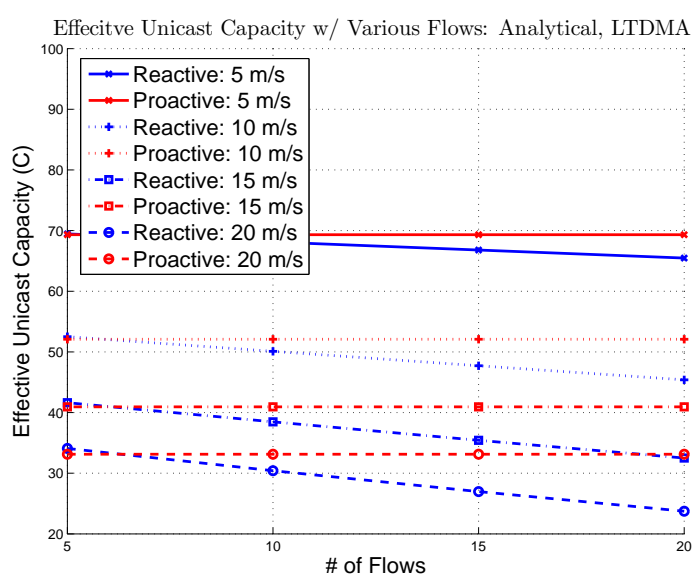

Fig. 4. Effective Unicast Capacity, Various Flows: Analytical, LTDMA.

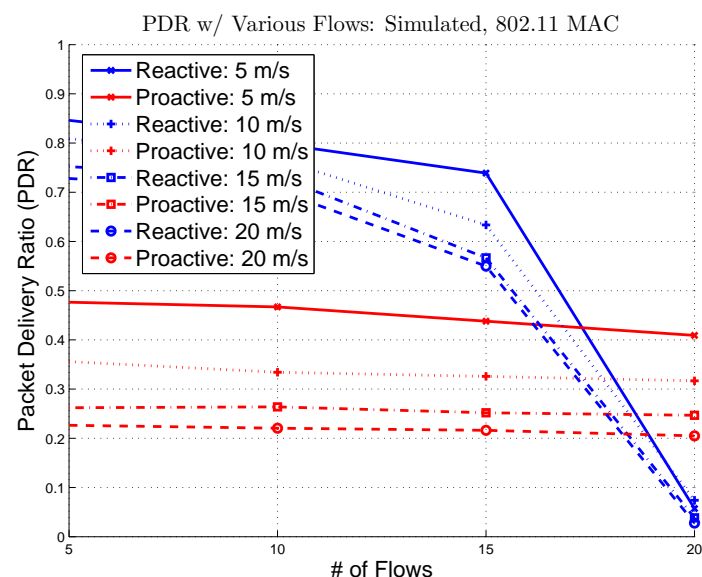

Fig. 6. Packet Delivery Ratio (PDR), Various Flows: Simulated, 802.11 MAC.

generating function as $C_{i}(z)=\left(1-p_{a}+p_{a} * z^{L}\right) * z$, where $p_{a}$ is the probability of packet arrivals from neighboring nodes at the same time slot. Clearly, it also corresponds to the packet collision probability of node $i$, i.e., $P_{e}=p_{a}$.

The packets competing with node $i$ consist of the sum of all traffic from neighboring nodes. The distribution of such arrival process can be approximated as Poisson, deduced from the superposition of random variables. Mathematically, the mean rate $\lambda_{i}^{c}$ of competing traffic can be written as

$$
\lambda_{i}^{c}=E\left(\sum_{\forall k \in\{\text { neighbors }\}}\left(\lambda_{B}^{k}+\lambda_{U}^{k}\right)\right)
$$

Then, the packet loss probability will be the probability of collision traffic arriving within a duration of a slot and can be computed as,

$$
\begin{aligned}
P_{e} & =\lambda_{i}^{c} * \eta \\
& \approx(N p-1)\left(\lambda_{B}+\lambda_{U}\right) * \eta
\end{aligned}
$$

where $\eta=20 \mu s$ in 802.11 DCF MAC.

We then look at the service aspect of M/G/1 model under such a MAC scheme. Let $\phi(z, L, \alpha, \gamma)$ be the probability generating function of service delay for each packet, where the 
collision probability is $\alpha$ and the back-off window value is $\gamma$. $\phi$ includes channel access time and the time needed to transmit the packet. The back-off counter value $M$ is uniformly chosen within $\gamma$ with the probability of $\frac{1}{\gamma}$.

Without collision, the total time to access the channel is the time needed for $M$ decreases, that is, $M$ times the busy time slot random variable $C_{i}$ which can be expressed by generating function $\sum_{i=1 \ldots \gamma} \frac{1}{\gamma} C_{i}(z)^{i}$. Once the channel is accessed the time needed to transmit the packet is fixed and equal to $L$. Therefore, it can be expressed by generating function $z^{L}$. Hence the service time when no collision occurs comes from adding the previous two quantities, or equivalently the corresponding generating function is equal to the product of the above generating functions, i.e.,

$$
\frac{z^{L}}{\gamma} \sum_{i=1 \ldots \gamma} C_{i}(z)^{i}=\frac{C_{i}(z)^{\gamma+1}-C_{i}(z)}{C_{i}(z)-1} \frac{z^{L}}{\gamma} .
$$

Eq.(34) is exactly the probability generating function of service time for broadcast packets, where packet collisions are not concerned. In case there is collision, the nodes select a new back-off number in a doubled contention window $\{1 \ldots 2 \gamma\}$ and the procedure is repeated which results in an additional service delay term. We obtain

$$
\begin{aligned}
\phi(z, L, \alpha, \gamma) & =\frac{C_{i}(z)^{\gamma+1}-C_{i}(z)}{C_{i}(z)-1} \frac{z^{L}}{\gamma} \\
& \times(1-\alpha+\alpha \phi(z, L, \alpha, 2 \gamma)) .
\end{aligned}
$$

Computing the probability generating function of service time through Eq.(35) for unicast packets requires a recursive computation, until the contention window length reaches the maximum value $C W_{\max }$.

Finally, we can summarize the probability generating function of service time for both broadcast packets $\phi_{B}(z)$ and unicast packets $\phi_{U}(z)$ as,

$$
\begin{aligned}
\phi_{B}(z) & =\frac{C_{i}(z)^{C W_{\min }+1}-C_{i}(z)}{C_{i}(z)-1} \frac{z^{L}}{C W_{\min }} \\
\phi_{U}(z) & =\phi\left(z, L, E\left(P_{e}^{i}\right), C W_{\min }\right)
\end{aligned}
$$

The mean service time for broadcast packets and unicast packets can then be computed as,

$$
\begin{aligned}
& \bar{S}_{B}=\left.\left(\frac{d}{d z} \phi_{B}(z)\right)\right|_{z=1} \\
& \mathcal{V}_{B}=\left(\left.\frac{d}{d z}\left(z * \frac{d}{d z} \phi_{B}(z)\right)\right|_{z=1}\right. \\
& \bar{S}_{U}=\left.\left(\frac{d}{d z} \phi_{U}(z)\right)\right|_{z=1} \\
& \mathcal{V}_{U}=\left(\left.\frac{d}{d z}\left(z * \frac{d}{d z} \phi_{U}(z)\right)\right|_{z=1}\right.
\end{aligned}
$$

\section{Simulations}

In the simulation, we consider a total of 100 nodes initially distributed randomly over a square network of size $1000 \mathrm{~m} \times 1000 \mathrm{~m}$. Three different transmission ranges $R \in$ $\{150,200,250\} m$ are covered, all within the coverage of WiFi devices. Four different speeds $V \in\{5,10,15,20\} \mathrm{m} / \mathrm{s}$ are simulated, from lower mobility to higher mobility scenarios.
Traffic, supplied from CBR source at rate $0.5 p / s$, is randomly generated with uniformly distributed sources and destinations. Different traffic flows $F \in\{1,5,10,15,20\}$ flows are simulated, covering low flow and moderate flow configurations. In addition, simulation results are obtained for both reactive (AODV [16]) protocol and proactive (OLSR [15]) protocol using the default implementation in Qualnet 3.9.5. The MAC layer is chosen as the default implementation of 802.11 MAC in Qualnet. Overall, a total of 120 different \{radius, mobility, flow, protocol $\}$ configurations are simulated. For each configuration, the simulation result is obtained from 10 random runs. Each simulation run is conducted at a randomly generated seed with a time duration of 30 minutes.

Figs. 3 and 4 present results of effective unicast capacity for scheduled TDMA MACs. The results clearly reflect the significant adverse effects from signaling overhead. The analytical results reveal that reactive protocols are more susceptible to traffic increase, while proactive protocols are robust to change in traffic. In general, proactive protocols are preferred in network profile of high traffic configuration, conforming the similar finding in [9] through control overhead analysis. The results also indicate that as mobility increases, performance of both protocols will be significantly affected. Eventually, at certain point, proactive protocols will completely cease to operate due to the increase in overhead, while reactive protocols could still operate but at very low traffic rate. Therefore, reactive protocols are favored in very high mobility scenarios.

We then explore the effectiveness of the proposed model in analyzing the general behaviors of routing protocols with more realistic 802.11 DCF MAC, in terms of packet delivery ratio (PDR), under various \{mobility, traffic flow $\}$ configurations. Note that when evaluating proactive protocols, the proposed model has been adapted to incorporate the analysis of OLSR protocol [14], accounting for artifacts from MPR technique. However, since there is no such analysis for AODV protocol, the generic reactive protocol described in Section II is used. Figs. 5 and 6 show that

- When tailored to specific protocols, the proposed model provides satisfactory approximation to simulated performance, as observed from good match between Fig. 5 and 6 for proactive (OLSR) protocol.

- Without incorporating specific techniques of AODV protocol (e.g. local repair), the proposed model still captures the essential behaviors of reactive protocols with respect to mobility and traffic flows, while failing to provide good matches to simulated performance.

It should be noted that although Fig. 6 only presents a small set of simulations, other obtained simulation results are similar and thus not presented. In summary, the parameterized analytical framework provides key insights into the compounding and interacting effects of network parameters, deeper understanding on essential protocol behaviors and capability of approximating practical performance with incorporation of protocol-specific techniques.

Utilizing the proposed model, we are now capable of investigating the effect from various network parameters. For example, we would like to know how the increase in transmission 


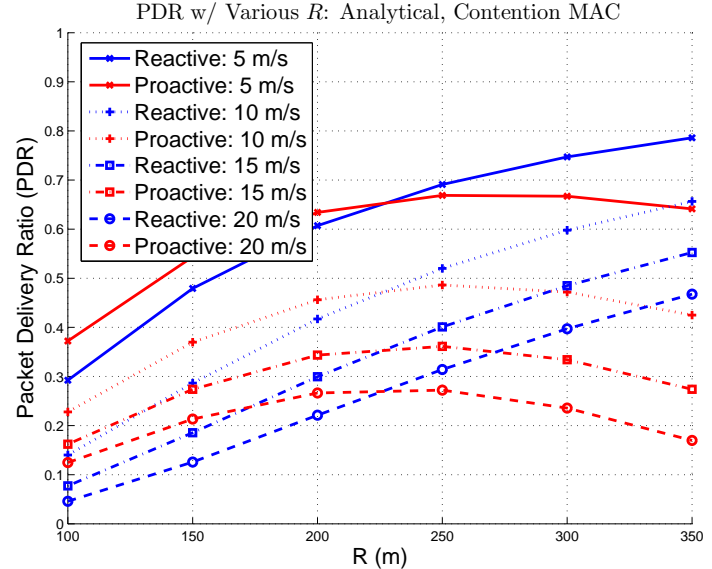

Fig. 7. Packet Delivery Ratio (PDR), Various R: Analytical, Contentionbased MAC.

radius $R$ affects protocol performance. Fig. 7 from the model immediately brings out the answer. The increase in $R$ results in two conflicting effects: improvements in signaling efficiency, resulting from the shorter source-destination distance; deteriorations in unicast capacity with more competing neighbor nodes. Furthermore, proactive protocols should expect worse performance due to the performance degradation of unicast capacity. These analytical result agrees well with our intuition. However, as presented in Fig. 8, our simulation being extensive but not comprehensive, still fails to capture such behavior. Clearly, our analytical model is essential not only to confirm and complement the simulations, but also to supply inherent clues to how changes in network parameters translate into performance variations.

\section{CONCLUSIONS}

We presented an analytical framework to evaluate the behavior of generic reactive and proactive protocols. In the model, the operation of the routing protocol is synthesized with the analysis of the MAC protocol to produce a parametric characterization of protocol performance. Corroborated from extensive simulations, the effectiveness and correctness of the model enable in-depth understanding of routing protocol performance.

\section{REFERENCES}

[1] J. Broch, D. A. Maltz, D. B. Johnson, Y. C. Hu, and J. Jetcheva. A performance comparison of multi-hop wireless ad hoc network routing protocols. In Proceedings of the 4th annual ACM/IEEE international conference on Mobile computing and networking, pages 85-97, New York, NY, USA, October 1998.

[2] S. Das, R. Castaneda, J. Yan, and R. Sengupta. Comparative performance evaluation of routing protocols for mobile. In The 7th International Conference on Computer Communications and Networks (IC3N), pages 153-161, Lafayette, Lousiana, USA, October 1998.

[3] P. Johansson, T. Larsson, N. Hedman, and B. Mielczarek. Scenario-based performance analysis of routing protocols for mobile ad-hoc networks. In Proceedings of 5th ACM/IEEE MOBICOM, pages 195-206, Seattle, Washington, USA, 1999.

[4] S. J. Lee, M. Gerla, and C. K. Toh. A simulation study of table-driven and on-demand routing protocols for mobile ad hoc networks. Network, IEEE, 13(4):48-54, 1999.

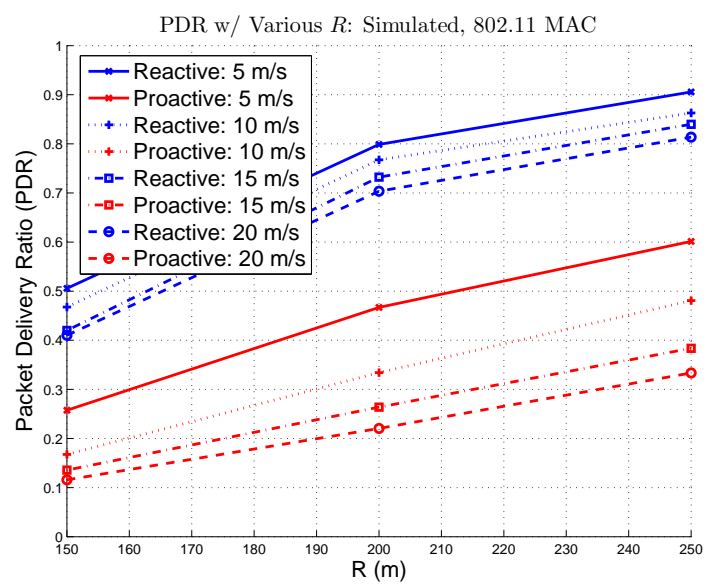

Fig. 8. Packet Delivery Ratio (PDR), Various $R$ : Simulated, 802.11 MAC.

[5] F. Bertocchi, P. Bergamo, G. Mazzini, and M. Zorzi. Performance comparison of routing protocols for ad hoc networks. In IEEE GLOBECOM, volume 2, pages 1033-1037, San Fransisco, California, USA, December 2003.

[6] A. Al-Maashri and M. Ould-Khaoua. Performance analysis of manet routing protocols in the presence of self-similar traffic. 31st IEEE Conference on Local Computer Networks, 0:801-807, January 2006.

[7] S. R. Das, C. E. Perkins, E. M. Royer, and M. K. Marina. Performance comparison of two on-demand routing protocols for ad hoc networks. IEEE Personal Communications Magazine special issue on Ad hoc Networking, 8(1):16-28, February 2001.

[8] N. Zhou, H. Wu, and A. A. Abouzeid. Reactive routing overhead in networks with unreliable nodes. In Mobicom'03, San Diego, September 2003.

[9] L. Viennot, P. Jacquet, and T. H. Clausen. Analyzing control traffic overhead versus mobility and data traffic activity in mobile ad-hoc network protocols. ACM/Springer Wireless Networks, 4(10):447-455, July 2004

[10] C. Bettstetter. Mobility modeling in wireless network: categorization, smooth movement and border effects. ACM Mobile Computing and Communication Review, 5:55-67, January 2001.

[11] W. Navid and T. Camp. Stationary distributions for the random waypoint model. IEEE Transactions on Mobile Computing, 3(1):99-108, 2004.

[12] J. Yoon, M. Liu, and B. Noble. Sound mobility models. In ACM Mobicom 2003, San Diego, 2003.

[13] J.-Y. Le Boudec and M. Vojnovic. Perfect simulation and stationarity of a class of mobility models. In INFOCOM 2005, Miami, 2005.

[14] X. Wu, H. R. Sadjadpour, and J. J. Garcia-Luna-Aceves. Modeling of topology evolutions and implication on proactive routing overhead in manets. Elsevier Computer Communications Journal, Special Issue on Algorithmic and Theoretical Aspects of Wireless Ad Hoc and Sensor Networks, 2007.

[15] T. Clausen and P. Jacquet. Optimized Link State Routing Protocol (OLSR). IETF RFC 3626, 2003.

[16] C. E. Perkins and E. M. Royer. Ad hoc on-demand distance vector routing. In Proc. of the 2nd IEEE Workshop on Mobile Computing Systems and Applications, pages 90-100, New Orleans, February 1999.

[17] G. Rodolakis. Analytical Models and Performance Evaluation in Massive Mobile Ad Hoc Networks - Chapter 2.2. PhD thesis, Ecole Polytechnique, 2006.

[18] L. Kleinrock. Queuing Systems, Volume 1: Theory. John Wiley \& Sons, Inc, 1975.

[19] X. Wu, H. R. Sadjadpour, and J. J. Garcia-Luna-Aceves. Link lifetime as a function of node mobility in manets with restricted mobility: Modeling and applications. In WiOpt 2007, April 2007.

[20] I. Rubin. Access-control disciplines for multi-access communication channels: Reservation and TDMA schemes. IEEE Transactions on Information Theory, 25(5):516-536, 1979.

[21] R. Nelson and L. Kleinrock. Spatial TDMA: A Collision-Free Multihop Channel Access Protocol. IEEE Transactions on Communications [legacy, pre-1988], 33(9):934-944, 1985.

[22] P. Gupta and P. R. Kumar. The capacity of wireless networks. IEEE Trans. on Information Theory, 46(2):388-404, March 2000. 